\title{
Persistence of protective immunity after postexposure prophylaxis of varicella with oral aciclovir in the family setting
}

\author{
Tetsushi Yoshikawa, Sadao Suga, Toru Kozawa, Shinji Kawaguchi, Yoshizo Asano
}

\begin{abstract}
The persistence of protective immunity after postexposure prophylaxis against varicella using oral aciclovir was evaluated in the family setting. Sixty one of 78 recipients of oral aciclovir were assessed by questionnaire, and 13 of 61 were evaluated for serum antibody to varicella zoster virus (VZV) using the fluorescent antibody to membrane antigen method. The observation period ranged from 33 to 50 months. None of those $(n=44)$ who had initially seroconverted to VZV after aciclovir prophylaxis developed breakthrough varicella. All 13 who had serology repeated still had titres $\geqslant 4$. Antibody titres in those who had histories of re-exposure to the virus were significantly higher than in those who had not $(\mathbf{p}<0.01)$. (Arch Dis Child 1998;78:61-63)
\end{abstract}

Keywords: aciclovir; postexposure prophylaxis; varicella; persistence of immunity

Varicella results from primary infection of a susceptible individual with varicella zoster virus (VZV). Although generally a mild disease, it can be complicated by bacterial superinfection of skin lesions, pneumonia, encephalitis, Reye's syndrome, or congenital varicella syndrome. The incidence of varicella is extremely high in children after exposure at home. Moreover, the clinical course in these children is generally more severe. ${ }^{1}$ It has been possible to prevent or modify the disease in the family setting by giving varicella zoster immune globulin, ${ }^{1}$ or live varicella vaccine, ${ }^{2}$ to children within 96 or 72 hours, respectively, of exposure. There has been, however, no procedure to prevent or modify the clinical course of varicella late in the incubation period. Recently, we reported successful prophylaxis with oral aciclovir after exposure late in the incubation period of varicella..$^{3-6}$ The efficacy of this procedure has been previously confirmed by other laboratories. ${ }^{7-9}$ In Japan, many parents would like to protect their children from natural varicella, so this method would be one option for preventing secondary cases of varicella after exposure at home. It is not clear, however, whether the patients who receive oral aciclovir prophylactically have the same degree of long term immunity as untreated patients. If immunity in recipients of prophylaxis wanes, then this procedure may create a population of adults who are at risk of serious illness. Furthermore, the question of whether these patients are more susceptible to zoster than patients who have natural varicella has not been answered. Four years ago, we gave oral aciclovir to a series of children to prevent secondary varicella in the family. In the present study, we evaluate the protective efficacy of, and the persistence of immunity to, VZV after oral aciclovir prophylaxis.

\section{Subjects and methods}

STUDY POPULATION

The subjects were 78 children ( 41 boys and 37 girls), who were treated prophylactically with oral aciclovir between January 1992 and July 1993 in our hospitals. The median age of children was 1.7 years (range 1 month to 9 years) at the time of aciclovir prophylaxis. All of the children were given orally between 20 and 80 $\mathrm{mg} / \mathrm{kg}$ daily of aciclovir in four divided doses for seven days, starting seven to nine days after exposure to the index case in their families. They were evaluated for immunity to VZV around two months after the prophylaxis.

CLINICAL FOLLOW UP

A letter was sent to parents of the children to explain the purpose of the study and to invite their child's participation. The letter also requested parents to bring their child to our hospitals to collect blood to test for the VZV antibody. Questionnaires sent to the parents, requested details relating to any instance of varicella or herpes zoster among their family, playmates, nursery school, school, or other settings. They were also asked whether their children had contracted breakthrough varicella or herpes zoster since they had received oral aciclovir prophylaxis. A telephone interview was conducted to clarify the information in several cases.

SEROLOGICAL EXAMINATION

Humoral immunity to VZV was examined by the fluorescent antibody to membrane antigen method. ${ }^{10}$

STATISTICAL ANALYSIS

Student's $t$ test was employed for statistical analysis. The statistical analysis was carried out using the logarithm of fluorescent antibody to membrane antigen titres.

\section{Results}

Replies to the questionnaire were obtained from 61 ( 35 boys and 26 girls) children. The median age of children was 1.8 years (range 3 months to 9 years) at the time of aciclovir prophylaxis. The 
Table 1 Clinical and immunological data of the 13 cases who had repeat serology approximately four years after aciclovir prophylaxis

\begin{tabular}{|c|c|c|c|c|c|c|c|}
\hline \multirow[b]{2}{*}{ Case No } & \multirow[b]{2}{*}{$\begin{array}{l}\text { Aget } \\
\text { (years) }\end{array}$} & \multirow[b]{2}{*}{ Sex } & \multirow[b]{2}{*}{$\begin{array}{l}\text { Dose of aciclovir } \\
\text { (mg/kg/day) }\end{array}$} & \multirow[b]{2}{*}{$\begin{array}{l}\text { Duration of } \\
\text { observation period } \\
\text { (months) }\end{array}$} & \multirow[b]{2}{*}{$\begin{array}{l}\text { Re-exposure of varicella } \\
\text { during the observation } \\
\text { period }\end{array}$} & \multicolumn{2}{|l|}{ Antibody titres to VZV } \\
\hline & & & & & & $\begin{array}{l}\text { At the time of convalescent } \\
\text { phase of aciclovir } \\
\text { prophylaxis }\end{array}$ & $\begin{array}{l}\text { Four years after } \\
\text { aciclovir } \\
\text { prophylaxis } \neq\end{array}$ \\
\hline 1 & 1 & $M$ & 80 & 50 & No & 16 & 4 \\
\hline 2 & 9 & $M$ & 80 & 46 & No & 8 & 8 \\
\hline 3 & 1 & $\mathrm{~F}$ & 20 & 45 & No & 8 & 8 \\
\hline 4 & 0.4 & $\mathrm{M}$ & 20 & 38 & No & $<4$ & 4 \\
\hline 5 & 3 & M & 20 & 35 & No & 256 & 4 \\
\hline 6 & 0.6 & $\mathrm{~F}$ & 5 & 33 & No & 8 & 8 \\
\hline 7 & 4 & $\mathrm{~F}$ & 18 & 45 & No & 128 & 4 \\
\hline $8^{\star}$ & 0.5 & $\mathrm{~F}$ & 40 & 45 & Yes & $<4$ & 8 \\
\hline 9 & 1 & $M$ & 20 & 37 & Yes & 64 & 8 \\
\hline $10^{\star}$ & 1 & $M$ & 40 & 38 & Yes & $<4$ & 16 \\
\hline 11 & 4 & $M$ & 30 & 48 & Yes & 16 & 16 \\
\hline 12 & 2 & $\mathrm{~F}$ & 18 & 45 & Yes & 8 & 16 \\
\hline $13^{\star}$ & 0.5 & $\mathrm{~F}$ & 40 & 47 & Yes & 8 & 64 \\
\hline
\end{tabular}

*These three cases showed an antibody rise during the observation period.

$\dagger$ Represents age at the time of aciclovir prophylaxis.

$\ddagger$ Antibody titres in cases 8 to 13 were significantly higher than those in cases 1 to 7 ( $<<0.01)$.

observation period ranged from 33 months to 50 months. None of the 61 subjects received immunosuppressive treatment during the observation period. Forty four (72\%) of the 61 recipients had had seroconversion against VZV after aciclovir prophylaxis. Moreover, these 44 recipients included 17 recipients who had presented with mild clinical features characterised by a lack of fever and a small number of skin lesions. Serology was carried out in 13 of the 61 cases at the end of the observation period.

None of the recipients who had an initial seroconversion against VZV after aciclovir prophylaxis developed breakthrough varicella during the observation period as part of this study. In total, 19 well documented contacts (three by siblings, 10 in nursery school, five by playmates, one in hospital) with patients who had varicella were reported. Despite these exposures, only two recipients (both of whom had contact with varicella in nursery school) developed varicella. Seroconversion against VZV was not documented in either of these two cases at the time of the convalescent phase of aciclovir prophylaxis. Both patients had approximately 100 vesicles on their trunk and had a high fever.

None of the group who had received aciclovir prophylaxis developed herpes zoster during this observation period.

Repeat serology was conducted in 13 of the 61 children who participated in the study (table). Each of the 13 individuals had an antibody titre of $\geqslant 4$ (ranging from 4 to 64 ). There was a history of exposure to VZV during the observation period in six of the 13 cases, and in a half of these there was a rise in their VZV antibody titres. Seroconversion was documented in cases eight and 10. Although both of the cases had a history of re-exposure to varicella during the observation period, they did not have clinical varicella at that time. Antibody titres in the recipients who had a history of re-exposure to VZV (case numbers 8 to 13) were significantly higher than in those recipients who were not re-exposed to the virus (case numbers 1 to 7$)(\mathrm{p}<0.01)$.

\section{Discussion}

Prophylaxis against varicella after exposure can be provided with varicella zoster immune globulin, ${ }^{1}$ live varicella vaccine, ${ }^{2}$ or aciclovir prophylaxis. Since our report on the efficacy of aciclovir prophylaxis in the family setting, ${ }^{3}$ there have been several further reports describing the benefits of this procedure.$^{7-9}$ However, two major concerns have been raised. One is whether recipients of prophylaxis have impaired protective immunity, and the other is whether the incidence of zoster is higher in this group. This study was designed to address the former question. The results obtained from this study are encouraging, although the numbers studied are limited. None of those who had seroconverted to VZV after aciclovir prophylaxis presented with breakthrough varicella, and all of those who had repeat serology at the end of the observation period had titres $\geqslant 4$, although the numbers studied were small. These results suggest that if the recipient acquires antibody to $\mathrm{VZV}$ after receiving aciclovir, then humoral immunity in the recipient will persist for at least three years. An assessment of the effects of aciclovir dose and clinical features at the time of the prophylaxis on the persistence of immunity against VZV would require a further prospective study controlled against patients not receiving aciclovir.

Since seroconversion to VZV is observed in only $84 \%$ of recipients of aciclovir prophylaxis, ${ }^{3}$ the remainder remain at risk of varicella. We therefore recommend that serology to $\mathrm{VZV}$ is checked in all aciclovir recipients and VZV vaccine given to those who do not seroconvert.

Those re-exposed to varicella during the observation period had significantly higher antibody titres than those who were not. Re-exposure to wild type varicella, which is still endemic in Japan, is likely to have been important in maintaining antibody titres to VZV in these children. It has been reported that anti-VZV antibody titres in children immunised with VZV vaccine increase with time after immunisation. ${ }^{10}$ It is possible that antibody titres to VZV among aciclovir prophylaxis recipients will also increase with time.

In this study, two recipients (case 8 and case 10) were found to have seroconverted to VZV during the observation period. Neither had a clinical history of varicella, but had been re-exposed. It is possible that they had a low 
concentration of VZV antibody during the convalescent phase of aciclovir prophylaxis which was not detected by our fluorescent antibody to membrane antigen test, and had protective immunity against breakthrough varicella. This suggests that better serological methods may be necessary to assess protection.

A longer and larger study would be necessary to assess the incidence of zoster in the recipients of aciclovir prophylaxis. Studies of immunocompromised children receiving aciclovir prophylaxis will also be important.

This work was supported in part by the grant from Fujita Health University.

1 Ross AH. Modification of chicken pox in family contacts by administration of gamma globulin. $N$ Engl $\mathcal{f} \mathrm{Med}$ 1962;267:369-76.

2 Asano Y, Nakayama H, Yazaki T, et al. Protection against varicella in family contacts by immediate inoculation with live varicella vaccine. Pediatrics 1977;59:3-7.
3 Asano Y, Yoshikawa T, Suga S, et al. Postexposure prophylaxis of varicella in family contact by oral acyclovir. Pediatrics 1993;92:219-22.

4 Suga S, Yoshikawa T, Ozaki T, et al. Effect of oral acyclovir against primary and secondary viraemia in incubation period of varicella. Arch Dis Child 1993;69:639-43.

5 Suga S, Yoshikawa T, Yazaki T, et al. Dose-dependent effects of oral acyclovir in the incubation period of varicella. Acta Paediatr 1996;85:1418-21.

6 Asano Y, Suga S, Yoshikawa T. Comparison of specific immunities to varicella-zoster virus following postexposure prophylaxis of varicella by oral acyclovir observed in a family. Acta Paediatr fpn 1996;38:692-4.

7 White CB, Hawley WZ, Harford DJ. The pediatric resident susceptible to varicella: providing immunity through postexposure prophylaxis with oral acyclovir. Pediatr Infect postexposure prophylaxi
Dis $\mathcal{F} 1994 ; 13: 743-5$.

8 Huang YC, Lin TY, Chiu CH. Acyclovir prophylaxis of varicella after household exposure. Pediatr Infect Dis $\mathcal{f}$ 1995;14:152-4.

9 Ishida Y, Tauchi H, Higaki A, et al. Postexposure prophylaxis of varicella in children with leukemia by oral acyclovir. Pediatrics 1996;97:150-1.

10 Asano Y, Albrecht P, Vujcic LK, et al. Five-year follow-up study of recipients of live varicella vaccine using enhanced neutralization and fluorescent antibody membrane antigen assays. Pediatrics 1983;72:291-4. 